\title{
Relación entre parasitosis intestinales y grado de educación sanitaria de la población
}

Birri, María L.*; Bezombe, Marta**; Manuale, Marcela**

\section{Resumen}

Se relacionó el grado de educación sanitaria de las madres del barrio marginal "Chaco Chico", Santa Fe , Argentina, con el nivel de infección y reinfección con enteroparásitos en sus hijos W de 14 años. La población fue analizada desde los aspectos socioeconómicos, de condiciones de vida y de asistencia médica, bioquímica y educativa. A pesar que la mayoría de las madres lograron aprobar el $7^{0}$ de la educación sistemática y que la población infantil está permanentemente asistida desde los aspectos médico y bioquímico, los niños se encuentran parasitados (94,34\%), poliparasitados (28\%) y reinfectados $(26,32 \%)$.

Del análisis de los datos muestrales se deduce la asociación entre la falta de educación sanitaria de las madres y la presencia de hijos para y poliparasitados. Lo que evidencia la ineficiencia de la escasa educación sanitaria y la carencia de una planificación de la instrucción con un abordaje interdisciplinario y permanente.

Palabras Clave: Enteroparasitosis - Educación Sanitaria - Atención Primaria de Salud. 